\title{
Glycemic Abnormalities in Adolescents
}

\author{
Ashraf Soliman ${ }^{1 *}$, Vincenzo De Sanctis ${ }^{2}$, Mohamed Yassin ${ }^{3}$, Rania Elalaily ${ }^{4}$ and Haidar H Almawashi ${ }^{5}$ \\ ${ }^{1}$ Departments of Pediatrics, Hamad General Hospital, Doha, Qatar \\ ${ }^{2}$ Pediatric and Adolescent Outpatient Clinic, Quisisana Hospital, Ferrara, Italy \\ ${ }^{3}$ Department of Hematology and Oncology Alamal Hospital, HMC, Doha, Qatar \\ ${ }^{4}$ Department of Primary Health Care (PHC), Doha, Qatar \\ ${ }^{5}$ Department of Pediatrics, AIWakra Hospital - HMC, Doha Qatar
}

\begin{abstract}
Glycemic abnormalities including prediabetes and Type 2 diabetes mellitus (T2DM) are emerging clinical problems in children and adolescents. Increased insulin resistance during pubertal development appears to uncover glycemic abnormalities. Pediatricians and physicians should be knowledgeable and conscious about the prevalence and progression of prediabetes to T2DM, the frequent mild or asymptomatic manifestation of T2DM in children and adolescents and the high risk of complications in these patients. Screening for glycemic abnormalities seems useful in high risk groups such as children and adolescents with obesity, family history of T2DM, and with clinical features suggestive of insulin resistance (hypertension, dyslipidemia, polycystic ovarian syndrome, or acanthosis nigricans). Modification of life style and reduction of obesity is still the prime target of prevention and early management of early glycemic abnormalities. However, use of hypoglycemic drugs is indicated when these measures fail to achieve adequate glycemic control.
\end{abstract}

Keywords: Adolescents; Diabetes; Prediabetes; Diagnosis; Monitoring

\section{Physiological Changes in Insulin Secretion and Glycemic Control in Healthy Adolescents}

During puberty insulin levels are higher compared to the prepubertal and post pubertal (adulthood) periods. Direct evidence of increased pubertal insensitivity to insulin has been proven using in vivo experiments. In healthy adolescents there is a decrease in insulinstimulated glucose uptake compared with pre-pubertal children. This puberty-related insensitivity to insulin is compensated by an increase in insulin secretion [1-3]. A large cross-sectional study of children and adolescents without diabetes found that insulin sensitivity was lowest at age 12 to 14 years (Tanner stage 3 of puberty) in both sexes, across all ethnic groups, returning to almost pre-pubertal levels in young people above 16 years of age (Tanner's stage 5). A significant correlation has been demonstrated between the speed of growth and an increase in fasting serum insulin in pubertal adolescents without diabetes [4-7].

The major hormonal changes that are associated with the onset of puberty include a two-fold increase in the secretion of Growth Hormone (GH), increase in insulin-like growth factor-I (IGF-I), and an increase in the sex steroids that lead to the remarkable increase in height, development of secondary sexual characteristics and change in body composition. The secretion of GH increases during puberty and declines after the pubertal growth spurt is completed. GH is known to be an important factor in reducing insulin sensitivity and insulinstimulated glucose metabolism has been correlated negatively with $\mathrm{GH}$ and/or IGF-I levels $[4,5]$.

The significance of these physiological changes in metabolism and insulin sensitivity for adolescents with type 2 and type 1 diabetes mellitus

1. Type 2 diabetes mellitus (T2DM) appears to be a substantial and growing problem among adolescents. Increased insulin insensitivity due to puberty as well as due to obesity in adolescents diagnosed with type 2 diabetes are important pathophysiologic mechanisms in all ethnic groups. Adolescents with T2DM2 have deterioration of glycemic control during this pubertal period [8-12].
2. In adolescents with Type $1 \mathrm{DM}$ (T1DM), it is now apparent that because of increased insulin insensitivity, insulin action decreases by between $30 \%$ and $50 \%$. Therefore, healthcare providers should be aware that insulin requirements increase by between $30 \%$ and $50 \%$ during puberty. If this fact is overlooked, an adolescent's blood glucose control and HbA1c levels will deteriorate [8-12].

\section{Prevalence of Prediabetes in Adolescents and Its Potential Risks}

Prediabetic states are prevalent among children and adolescents. The prevalence of prediabetes depends on the definition used. Significant differences in prevalence can depend on whether prediabetes is defined by Impaired Fasting Glucose (IFG), Impaired Glucose Tolerance (IGT), or using $\mathrm{HbAlC}$ criteria as well as considering the age and ethnicity of the patients. In adolescents, the prevalence of IFG ranges from $13 \%$ to $40 \%$ and of IGT, from $2 \%$ to $5 \%$, whereas approximately $20 \%$ have $\mathrm{HbAlc}$ levels of $5.7 \%-6.4 \%$. The prevalence of prediabetes using IFG and IGT (in 2005-2006) among adolescents in the US aged 12-19 years, using the ADA criteria, showed that $13.1 \%$ had IFG, $3.4 \%$ had IGT and $16.1 \%$ had both IFG and IGT [13]. Moreover, the prevalence of prediabetes has increased markedly over recent decades [14,15] Among 3,400 adolescents in the US aged 12 to 19 (from the NHANES survey) the prevalence of prediabetes/diabetes increased significantly, from 9 percent to 23 percent between 1999 and 2008 [16].

*Corresponding author: Ashraf $\mathrm{T}$ Soliman, Professor of Pediatrics and Endocrinology, Alexandria, University Children's Hospital, Alexandria, Egypt, Tel: 00974-55983874; E-mail: ATSOLIMAN@yahoo.com

Received August 28, 2014; Accepted September 29, 2014; Published October 07,2014

Citation: Soliman A, De Sanctis V, Yassin M, Elalaily R, Haidar H, et al. (2014) Glycemic Abnormalities in Adolescents. J Diabetes Metab 5: 448 doi:10.4172/21556156.1000448

Copyright: (c) 2014 Soliman A, et al. This is an open-access article distributed under the terms of the Creative Commons Attribution License, which permits unrestricted use, distribution, and reproduction in any medium, provided the original author and source are credited. 
The prevalence of prediabetes is significantly higher among obese pubertal children with a positive family history of T2DM. In the Studies to Treat or Prevent Pediatric Type 2 Diabetes (STOPPT2D), conducted in 2003 in 8th-grade students (mean age, 13.6 years), $40.5 \%$ had fasting glucose levels $\geq 100 \mathrm{mg} / \mathrm{dL}$. In those with a body mass index (BMI) at the 95th percentile or higher, the prevalence increased to $47 \%$. $36.5 \%$ of students with normal BMI (< 85th percentile) also had fasting glucose levels $\geq 100 \mathrm{mg} / \mathrm{dL}$ [17]. In a multiethnic cohort study conducted between 2005 and 2010, 1156 obese children and adolescents (40\% boys) underwent an Oral Glucose Tolerance Test (OGTT) and HbAlc measurement. In 21\% of these patients, HbA1c levels were 5.7\%-6.4\%. Among children classified as being at increased risk for diabetes on the basis of their $\mathrm{HbAlc}$ values, only $47 \%$ were categorized as being at increased risk on the basis of their OGTT results. Moreover, 27\% of children with $\mathrm{HbA1c}$ levels below $5.7 \%$ were diagnosed with prediabetes on the basis of OGTT results [18].

Agreement is poor between $\mathrm{HbA1c}$ levels and OGTT results. It is not clear which of these measures will more closely predict long-term complications. HbAlc alone is a poor diagnostic tool for prediabetes in obese children and adolescents. The ADA panel, when recommending $\mathrm{HbA} 1 \mathrm{c}$ for diagnosis in adults, acknowledged that concordance is not perfect among $\mathrm{HbAlc}$, fasting plasma glucose, and 2-hour fasting plasma glucose levels. Nevertheless, long-term data on $\mathrm{HbA1c}$ elevation and risk for retinopathy, combined with practical considerations on the use of HbA1c, led the ADA to recommend its use $[19,20]$.

Among adults, prediabetes is a recognized risk factor for both T2DM and cardiovascular disease and is associated with retinopathy, a 2-fold increased incidence of micro albuminuria, and neuropathy. Concerns have been raised about the potential effect of prediabetes on morbidity in adolescents. However, no available data that describes the impact of prediabetes on morbidity in children [20-22].

\section{Identified Risk Factors for Prediabetes}

Obesity, family history of diabetes especially parental diabetes and male sex pose high risk rate of prediabetes. Adolescents aged 12-15 years have a significantly higher rate of prediabetes than those aged 16-19 years. This may be related to the peak of pubertal insulin resistance that occurs during early adolescence. Adolescents with 2 or more cardio metabolic risk factors (hypertension, dyslipidemia, and hypertriglyceridemia) have a 2.7 -fold higher rate of prediabetes than those with no cardio-metabolic risk factors. Similarly, adolescents with hyper-insulinemia have a 4 -fold higher prevalence than those without cardio-metabolic risk factors [23-27].

\section{The Fate of Prediabetes and Predictors of Progression from Prediabetes to T2DM?}

The story of developing T2DM from normal glucose tolerance entails many successive stages. The first stage is characterized by insulin resistance associated with a compensatory increase in insulin secretion; this stage can take several years. Patients with both IFG and IGT have insulin resistance, but the site of their predominant insulin resistance differs [28]. Those with IFG have predominantly hepatic insulin resistance, whereas those with IGT have predominantly muscle insulin resistance. In the second stage, where beta-cells fail to compensate for increased insulin resistance, hyperglycemia develops progressing from prediabetes to overt diabetes. This progressive loss of beta-cell secretion probably has a combination of genetic, environmental, and biochemical determinants. In children and adolescents, the early appearance of prediabetes and T2DM suggests an accelerated pathophysiologic process in the young $[16,29]$.

In a study of 117 obese children and adolescents (mean age: 12.7 years), OGTT tests were performed at baseline and after approximately 2 years out of 33 children with IGT at baseline, $45.5 \%$ reverted to normal glucose tolerance, $30.3 \%$ continued to have IGT, and $24.2 \%$ developed T2DM. Severe obesity, weight gain, IGT, and black race appeared as the best predictors of developing T2DM, whereas baseline fasting glucose, insulin level, and C-peptide level were not predictive [30]. In another study, 128 overweight/obese Hispanic children with a family history of T2DM were evaluated annually for 4 years with an OGTT (the ADA criteria for prediabetes were used). Tremendous variability was seen in the individual patterns of change in glucose status from year to year. Only $13 \%$ of participants had persistent prediabetes, whereas $47 \%$ had intermittent prediabetes, with no clear pattern over time [31]

A high rate of reversion from IGT to normal glucose tolerance was demonstrated in 128 obese white European adolescents (mean age: 13.5 years) diagnosed with IGT in 2003-2006. After a mean of 3.9 years of follow-up, $75 \%$ of these adolescents converted to normal glucose metabolism, $16 \%$ continued to have IGT, $2 \%$ developed T2DM, and 7\% were lost to follow-up. Age, sex, BMI, blood pressure, and fasting glucose levels at baseline did not differ between adolescents who continued to have IGT and those who converted to normal glucose metabolism. However, 2-hour glucose levels by OGTT at baseline were significantly higher in the adolescents who continued to have IGT and were highest in adolescents who developed diabetes. These studies demonstrate that a reasonably high percentage of children and adolescents diagnosed with prediabetes revert to normal glucose tolerance [32].

\section{Prediabetes and Type 1 Diabetes}

Prediabetes was defined to identify patients at risk forT2 DM however, IFG, elevated HbA1C, and IGT also can be the first signs of T1DM. The clinical presentation of T1DM is preceded by an asymptomatic latent period, and because obese adolescents can also develop T1DM, screening of obese patients for prediabetes might identify such cases. Assessment of diabetes-associated autoantibodies might be helpful. This prediabetic period can last for months or years [20].

\section{Potential Treatment Options and Screening for Prediabetes}

The expert panel of the ADA focusing on prediabetic states acknowledged the strong association between diabetes and obesity and suggested that the first priority is maintenance of a healthy weight and prevention of obesity [20]. Although several studies have demonstrated the success of interventions to prevent obesity in children and adolescents, no studies have shown how to prevent the progression from prediabetes to diabetes in this age group. Longitudinal studies are required to document these outcomes.

Even though adoption of a healthy lifestyle is beneficial to everyone; however, the question of drug therapy in children and adolescents is much more complicated. Several short-term studies (about 6 months in duration) in normoglycemic adolescents at risk for diabetes as a result of elevated BMI, family history, and elevated fasting insulin level have shown that compared with placebo, treatment with metformin results in a progressive decline in fasting blood glucose and a reduction in fasting insulin levels [33-36]. In a meta-analysis of 4 randomized controlled trials in participants younger than 19 years who were treated with metformin for at least 2 months, a statistically significant mean 
reduction in fasting insulin and BMI was seen with metformin (with and without lifestyle intervention) compared with placebo. However, because of small size and short duration of these studies none was able to document prevention of progression to diabetes [37]. Metformin treatment of 15 obese adolescents with the polycystic ovary syndrome and IGT was found to be beneficial and after 3 months 8 patients had normal glucose tolerance [38].

In June 2009, an International Committee composed of experts from the American Diabetes Association, the European Association for the Study of Diabetes and the International Diabetes Federation recommended that prediabetes testing includes the $\mathrm{Hb} \mathrm{AlC}$ test, fasting blood sugar test and oral glucose tolerance test [39]. According to the American Diabetes Association recommendations it would be helpful to consider monitoring patients with impaired fasting glucose or HbAlc (5.7\%-6.4\%) or previously Impaired Fasting Glucose (IFG) every year. Monitoring should include at least annual reassessment of FPG and/or an OGTT. For individuals where progression is suspected, annual measurements of FPG and HbA1C, with 2-hour OGTT, should all be conducted.

The AACE recommends $\mathrm{HbA1C}$ primarily for screening and monitoring and not for prediabetes identification. This is because the A1C test can be misleading or inaccurate in some populations [38-44].

In summary, screening for prediabetes and monitoring of glycemic abnormalities seems to be meaningful in adolescents who have a parental history of diabetes, are extremely obese, and have entered puberty; especially in adolescents of certain ethnic groups.

\section{The Prevalence of T2DM in Children and Adolescents}

The difficulties to detect T2DM in children and adolescents include a) Hyperglycemia can go undiagnosed for a long time, b) it may have no symptoms or mild symptoms, c) blood tests of variable sensitivity are needed for diagnosis d) criteria for differentiating between T1DM and T2DM in children and adolescents may be confusing. Children and adolescents with T2DM can develop ketoacidosis; while those with T1DM can be overweight; and therefore the overall prevalence of the disease may still be low. This means that scientists will have to sample a very large population of adolescents in order to find a stable estimate of prevalence [12].

T2DM appears to be an evolving as a new clinical problem within pediatric practice. Recent reports indicate an increasing prevalence of type 2 diabetes mellitus in children and adolescents around the world in all ethnicities, even if the prevalence of obesity is not increasing any more. Ethnic subgroups such as African-American, Hispanic, Asian/Pacific Islanders and American Indians pose a higher risk to develop T2DM in adolescents. The frequent mild or asymptomatic manifestation of T2DM in children and adolescents proposes that screening is important especially in high risk groups such as children and adolescents with obesity, relatives with type 2 diabetes mellitus, and clinical features of insulin resistance (hypertension, dyslipidemia, polycystic ovarian syndrome, or acanthosis nigricans) [45].

Adolescents with T2DM appear to be at a much higher risk of developing early diabetes mellitus associated complications than those with T1DM. This higher level of risk does not appear to be related to overall levels of glycemic control or duration of disease but to occurrence of hypertension and dyslipidemia [46,47].

Treatment of choice is lifestyle intervention followed by pharmacological treatment (e.g., metformin). New drugs such as dipeptidyl peptidase inhibitors or glucagon like peptide 1 mimetics are still under trials for treatment of youth with T2DM. Since microvascular complications of T2DM like retinopathy and nephropathy already occur in children, dilated eye examinations should be performed and screening for microalbuminuria should also be performed yearly. Angiotensin Converting Enzyme (ACE) inhibitors are the agents of choice in children with microalbuminuria. Recent reports also indicate a significant failure of the medical care system of adolescents withT2 DM suggesting and recommending remodeling of current healthcare practices $[45,48]$.

In 107 patients with clinically diagnosed T2DM (aged $\leq 20 \mathrm{yr}$ at diagnosis), patients were obese (BMI -SDS): 2.6 (2.0-3.1)], and $88.0 \%$ had a family history of diabetes. Islet autoantibodies were detected in (15.0\%). Autoantibody positive patients had poorer metabolic control than autoantibody negative patients. Therapies used were lifestyle only (35.5\%), oral anti-diabetics (OAD) only (43.3\%), insulin + OAD (15.9\%) and insulin only (5.6\%). Complications including metabolic syndrome (61.7\%), microalbuminuria (13.4\%), and retinopathy $(3.9 \%)$ were diagnosed. Patients with $\beta$-cell autoimmunity or HLA-DR genetic risk more frequently used insulin than confirmed T2DM patients $(50.0$ vs. $22.0 \%)[49]$.

\section{Screening for T2DM in Adolescents}

The Canadian Diabetic Association recommends that screening for T2DM should be performed every 2 years using an fasting plasma glucose test (FPG test) in children and adolescents with any of the following [50]:

1. $\geq 3$ risk factors in non-pubertal or $\geq 2$ risk factors in pubertal children

2. Obesity (BMI $\geq 95$ th percentile for age and gender)

3. Member of a high-risk ethnic group (e.g. Aboriginal, African, Asian, Hispanic or South Asian descent)

4. Family history of $\mathrm{T} 2 \mathrm{DM}$ and/or exposure to hyperglycemia in utero

5. Signs or symptoms of insulin resistance (including acanthosis nigricans, hypertension, dyslipidemia, Non-Alcoholic Fatty Liver Disease (NAFLD) (ALT: 3 times upper limit of normal or fatty liver on ultrasound) and features of polycystic ovary disease (PCOS).

The ADA recommends a screening in overweight children and adolescents at onset of puberty in high risk patients (BMI $>90$ percentile + one of the followings; family history of T2DM, Asian, American Indian, Africa-Americans or Hispanic race, acanthosis nigricans, hypertension, dyslipidemia or polycystic ovarian syndrome). Testing is recommended every 2 years starting at the age of 10 years or at onset of puberty if it occurs in a younger age by fasting glucose or OGTT [48].

Screening in those high- risk groups demonstrated T2DM in around $1 \%$ of obese Caucasian children in Germany and in $4 \%$ of screened obese adolescents in particular ethnic groups in the United States $[51,52]$.

\section{Conclusion}

Prediabetic states are prevalent among children and adolescents. Moreover, the prevalence of prediabetes has increased markedly over recent decades. Screening for prediabetes and monitoring of glycemic abnormalities seems to be meaningful in adolescents who have a parental history of diabetes, are extremely obese, especially in 
Citation: Soliman A, De Sanctis V, Yassin M, Elalaily R, Haidar H, et al. (2014) Glycemic Abnormalities in Adolescents. J Diabetes Metab 5: 448 doi:10.4172/2155-6156.1000448

Page 4 of 5

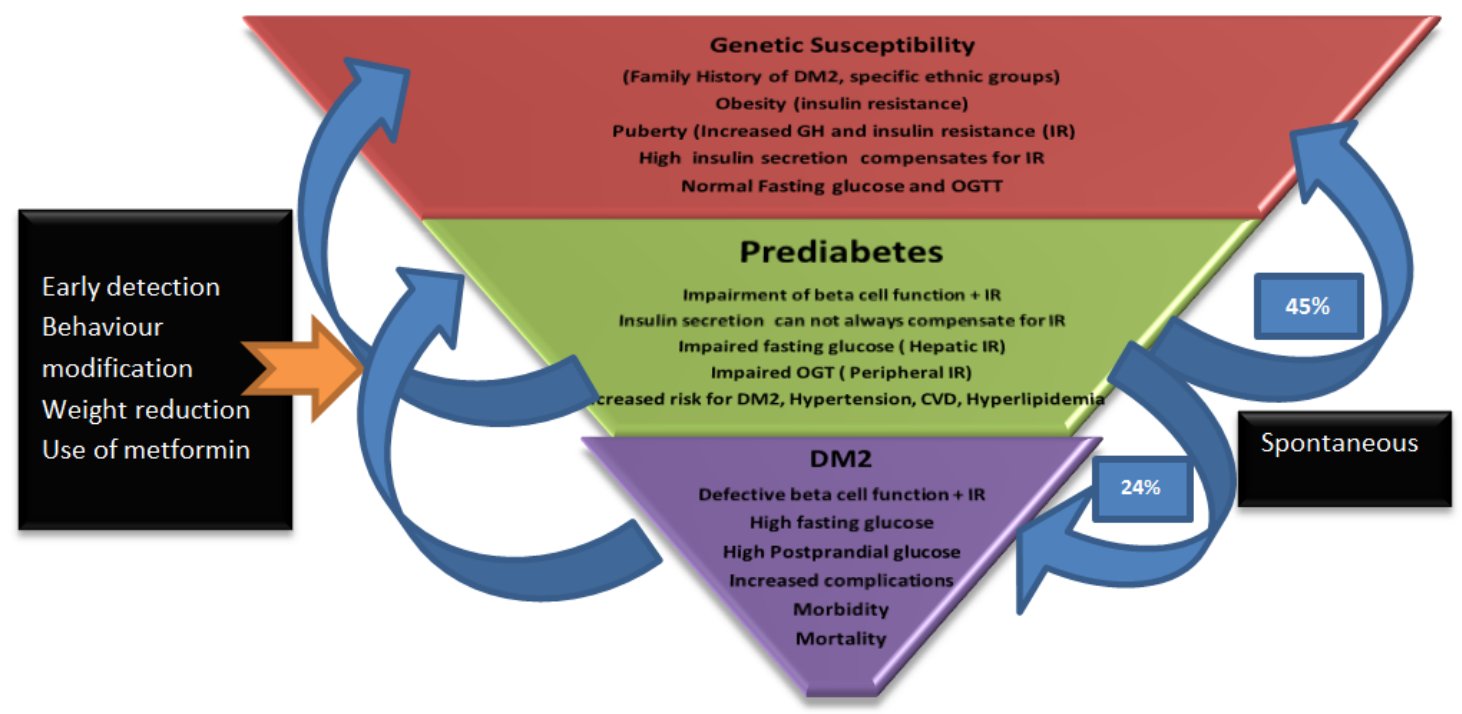

Figure 1: Sequence of type 2 diabetes mellitus development in adolescents and its reversibility.

adolescents of certain ethnic groups and affected by special systemic diseases with potential insult to the beta cells. Recent reports indicate an increasing prevalence of T2DM prevalence in adolescents all over the world possibly due to increasing obesity. Obese adolescents with clinical signs of insulin resistance (acanthosis nigricans, dyslipidemia, hypertension, PCOS) or family history of T2DM and those with certain ethnicity have higher risk to develop T2DM. It seems appropriate to screen those children and adolescents above the age of 10 years for T2DM. Early detection, prevention and treatment of prediabetes and T2DM should become one of the targets of public health intervention programs. Physicians should make the public aware of both adolescent obesity epidemic and its serious consequences including prediabetes and T2DM (Figure 1).

\section{References}

1. Klonoff DC (2005) Continuous glucose monitoring: roadmap for 21st century diabetes therapy. Diabetes Care 28: 1231-1239.

2. Amiel SA, Sherwin RS, Simonson DC, Lauritano AA, Tamborlane WV (1986) Impaired insulin action in puberty. A contributing factor to poor glycemic control in adolescents with diabetes. N Engl J Med 315: 215-219.

3. Caprio S, Plewe G, Diamond MP, Simonson DC, Boulware SD, et al. (1989) Increased insulin secretion in puberty: a compensatory response to reductions in insulin sensitivity. J Pediatr 114: 963-967.

4. Moran A, Jacobs DR Jr, Steinberger J, Cohen P, Hong CP, et al. (2002) Association between the insulin resistance of puberty and the insulin-like growth factor-I/growth hormone axis. J Clin Endocrinol Metab 87: 4817-4820.

5. Arslanian SA, Kalhan SC (1994) Correlations between fatty acid and glucose metabolism. Potential explanation of insulin resistance of puberty. Diabetes 43 : 908-914

6. Hannon TS, Janosky J, Arslanian SA (2006) Longitudinal study of physiologic insulin resistance and metabolic changes of puberty. Pediatr Res 60: 759-763.

7. Tfayli H, Arslanian S (2007) The challenge of adolescence: hormonal changes and sensitivity to insulin. Diabetes Voice 52: 28-30

8. Writing Group for the SEARCH for Diabetes in Youth Study Group1, Dabelea D, Bell RA, D'Agostino RB Jr, Imperatore G, et al. (2007) Incidence of diabetes in youth in the United States. JAMA 297: 2716-2724.

9. Anonymous.Concern as spike in type 1 diabetes is seen in US youth. The Wall Street Journal

10. Mortensen HB, Robertson KJ, Aanstoot HJ, Danne T, Holl RW, et al. (1998)
Insulin management and metabolic control of type 1 diabetes mellitus in childhood and adolescence in 18 countries. Hvidore Study Group on Childhood Diabetes. Diabet Med 15: 752-759.

11. Type 2 diabetes in children and adolescents. American Diabetes Association. Diabetes Care 23: 381-389.

12. Anonymous Epidemiology of Type 1 and Type 2 Diabetes Mellitus Among North American Children and Adolescents (2013) Center for Diabetes Control and Prevention.

13. Li C, Ford ES, Zhao G, Mokdad AH (2009) Prevalence of pre-diabetes and its association with clustering of cardiometabolic risk factors and hyperinsulinemia among U.S. adolescents: National Health and Nutrition Examination Survey 2005-2006. Diabetes Care 32: 342-347.

14. Expert Committee on the Diagnosis and Classification of Diabetes Mellitus (2003) Report of the expert committee on the diagnosis and classification of diabetes mellitus. Diabetes Care 26: S5-20.

15. Balion CM, Raina PS, Gerstein HC, Santaguida PL, Morrison KM, et al. (2007) Reproducibility of impaired glucose tolerance (IGT) and impaired fasting glucose (IFG) classification: a systematic review. Clin Chem Lab Med 45: 11801185.

16. Weiss $R$ (2007) Impaired glucose tolerance and risk factors for progression to type 2 diabetes in youth. Pediatr Diabetes 8 Suppl 9: 70-75.

17. Baranowski T, Cooper DM, Harrell J, Hirst K, Kaufman FR, et al. (2006) Presence of diabetes risk factors in a large U.S. eighth-grade cohort. Diabetes Care 29: 212-217.

18. Nowicka P, Santoro N, Liu H, Lartaud D, Shaw MM, et al. (2011) Utility of hemoglobin $\mathrm{A}(1 \mathrm{c})$ for diagnosing prediabetes and diabetes in obese children and adolescents. Diabetes Care 34: 1306-1311.

19. Guerrero-Romero F, Violante R, Rodriguez-Moran M (2009) Distribution of fasting plasma glucose and prevalence of impaired fasting glucose, impaired glucose tolerance and type 2 diabetes in the Mexican paediatric population. Paediatr Perinat Epidemiol 23:363-369.

20. Pinhas-Hamiel O, Zeitler P. Prevalence of Prediabetes in the Pediatric Population. Prediabetes in Children and Adolescents: What Does It Mean?

21. Grundy SM (2012) Pre-diabetes, metabolic syndrome, and cardiovascular risk J Am Coll Cardiol 59: 635-643.

22. Nathan DM, Davidson MB, DeFronzo RA, Heine RJ, Henry RR, et al. (2007) American Diabetes Association. Impaired fasting glucose and impaired glucose tolerance: implications for care. Diabetes Care 30:753-759.

23. Li C, Ford ES, Zhao G, Mokdad AH (2009) Prevalence of pre-diabetes and its association with clustering of cardiometabolic risk factors and hyperinsulinemia 
Citation: Soliman A, De Sanctis V, Yassin M, Elalaily R, Haidar H, et al. (2014) Glycemic Abnormalities in Adolescents. J Diabetes Metab 5: 448 doi:10.4172/2155-6156.1000448

Page 5 of 5

among U.S. adolescents: National Health and Nutrition Examination Survey 2005-2006. Diabetes Care 32: 342-347.

24. Cambuli VM, Incani M, Pilia S, Congiu T, Cavallo MG, et al. (2009) Oral glucose tolerance test in Italian overweight/obese children and adolescents results in a very high prevalence of impaired fasting glycaemia, but not of diabetes. Diabetes Metab Res Rev 25:528-534.

25. Rodriguez-Moran M, Guerrero-Romero F, Aradillas-Garcia C, Violante $R$ Simental-Mendia LE, et al. (2010) Obesity and family history of diabetes as risk factors of impaired fasting glucose: implications for the early detection of prediabetes. Pediatr Diabetes 11:331-336.

26. Reinehr T, Wabitsch M, Kleber M, de Sousa G, Denzer C, et al. (2009) Parental diabetes, pubertal stage, and extreme obesity are the main risk factors for prediabetes in children and adolescents: a simple risk score to identify children at risk for prediabetes. Pediatr Diabetes 10:395-400.

27. Weiss R, Taksali SE, Tamborlane WV, Burgert TS, Savoye M, et al. (2005) Predictors of changes in glucose tolerance status in obese youth. Diabetes Care 28: 902-909.

28. Tabák AG, Herder C, Rathmann W, Brunner EJ, Kivimäki M (2012) Prediabetes: a high-risk state for diabetes development. Lancet 379: 2279-2290.

29. May AL, Kuklina EV, Yoon PW (2012) Prevalence of cardiovascular disease risk factors among US adolescents, 1999-2008. Pediatrics 129: 1035-1041.

30. Weiss R (2007) Impaired glucose tolerance and risk factors for progression to type 2 diabetes in youth. Pediatr Diabetes 8 Suppl 9: 70-75.

31. Goran MI, Lane C, Toledo-Corral C, Weigensberg MJ (2008) Persistence of pre-diabetes in overweight and obese Hispanic children: association with progressive insulin resistance, poor beta-cell function, and increasing visceral fat. Diabetes 57: 3007-3012.

32. Kleber M, deSousa G, Papcke S, Wabitsch M, Reinehr T (2011) Impaired glucose tolerance in obese white children and adolescents: three to five year follow-up in untreated patients. Exp Clin Endocrinol Diabetes 119: 172-176.

33. Freemark M, Bursey D (2001) The effects of metformin on body mass index and glucose tolerance in obese adolescents with fasting hyperinsulinemia and a family history of type 2 diabetes. Pediatrics 107: E55.

34. Kay JP, Alemzadeh R, Langley G, D'Angelo L, Smith P, et al. (2001) Beneficial effects of metformin in normoglycemic morbidly obese adolescents. Metabolism 50: 1457-1461.

35. Srinivasan S, Ambler GR, Baur LA, Garnett SP, Tepsa M, et al. (2006) Randomized, controlled trial of metformin for obesity and insulin resistance in children and adolescents: improvement in body composition and fasting insulin. $\mathrm{J}$ Clin Endocrinol Metab 91: 2074-2080.

36. Yanovski JA, Krakoff J, Salaita CG, McDuffie JR, Kozlosky M, et al. (2011) Effects of metformin on body weight and body composition in obese insulinresistant children: a randomized clinical trial. Diabetes 60: 477-485.

37. Quinn SM, Baur LA, Garnett SP, Cowell CT (2010) Treatment of clinical insulin resistance in children: a systematic review. Obes Rev 11: 722-730.

38. Arslanian SA, Lewy V, Danadian K, Saad R (2002) Metformin therapy in obese adolescents with polycystic ovary syndrome and impaired glucose tolerance: amelioration of exaggerated adrenal response to adrenocorticotropin with reduction of insulinemia/insulin resistance. J Clin Endocrinol Metab 87: 15551559
39. American Diabetes Association (2010) Executive Summary - Standards of Medical Care in Diabetes. Diabetes Care 33: S4-S10.

40. American Diabetes Association (2013) Standards of medical care in diabetes--2013. Diabetes Care 36 Suppl 1: S11-66.

41. Goldberg RM, Cheng AYY, Punthaker Z, Clement M (2010) Use of glycated hemoglobin $(\mathrm{A} 1 \mathrm{C})$ in the diagnosis of type 2 diabetes mellitus in adults. Can $\mathrm{J}$ Diabetes 35:247-249.

42. Handelsman Y, Mechanick JI, Blonde L, Grunberger G, Bloomgarden ZT, et al. (2011) AACE Task Force for Developing Diabetes Comprehensive Care Plan. American Association of Clinical Endocrinologists medical guidelines for clinical practice for developing a diabetes mellitus comprehensive care plan. Endocr Pract 17: 1-53.

43. American Diabetes Association (2013) Standards of medical care in diabetes--2013. Diabetes Care 36 Suppl 1: S11-66.

44. Garber AJ , Handelsman Y, Einhorn D , Bergman DA, Bloomgarden ZT, et al. (2008) Diagnosis and management of prediabetes in the continuum of hyperglycemia: when do the risks of diabetes begin? A consensus statement from the American College of Endocrinology and the American Association of Clinical Endocrinologists. Endocr Pract. 14:933-946.

45. Reinehr T (2013) Type 2 diabetes mellitus in children and adolescents. World J Diabetes 4: 270-281.

46. Eppens MC, Craig ME, Cusumano J, Hing S, Chan AK, et al. (2006) Prevalence of diabetes complications in adolescents with type 2 compared with type 1 diabetes. Diabetes Care 29: 1300-1306.

47. Copeland KC, Zeitler P, Geffner M, Guandalini C, Higgins J, et al. (2011) Characteristics of adolescents and youth with recent-onset type 2 diabetes: the TODAY cohort at baseline. J Clin Endocrinol Metab 96: 159-167.

48. Type 2 diabetes in children and adolescents. American Diabetes Association Diabetes Care 23: 381-389.

49. Awa WL, Boehm BO, Rosinger S, Achenbach P, Ziegler AG, et al. (2013) HLA-typing, clinical, and immunological characterization of youth with type 2 diabetes mellitus phenotype from the German/Austrian DPV database. Pediatr Diabetes 14:562-574

50. Canadian Diabetes Association Clinical Practice Guidelines Expert Committee Panagiotopoulos C, Riddell MC, Sellers EA (2013) Type 2 diabetes in children and adolescents. Can J Diabetes 37 Suppl 1: S163-167.

51. Wabitsch M, Hauner H, Hertrampf M, Muche R, Hay B, et al. (2004) Type II diabetes mellitus and impaired glucose regulation in Caucasian children and adolescents with obesity living in Germany. Int J Obes Relat Metab Disord 28 : 307-313.

52. Sinha R, Fisch G, Teague B, Tamborlane WV, Banyas B, et al. (2002) Prevalence of impaired glucose tolerance among children and adolescents with marked obesity. N Engl J Med 346: 802-810. 